\title{
Efficacy and safety of nelfinavir in asymptomatic and mild COVID-19 patients: a structured summary of a study protocol for a multicenter, randomized controlled trial
}

Naoki Hosogaya ${ }^{1,2}$, Taiga Miyazaki ${ }^{2,3^{*}}$ (D), Yuri Fukushige ${ }^{1,4}$, Sachiko Takemori ${ }^{1}$, Shinpei Morimoto', Hiroshi Yamamoto ${ }^{1}$, Makoto Hori ${ }^{5}$, Tomoya Kurokawa ${ }^{5}$, Yohei Kawasaki ${ }^{5}$, Michiko Hanawa ${ }^{5}$, Yasuhisa Fujii ${ }^{5}$, Hideki Hanaoka ${ }^{5}$, Shingo Iwami ${ }^{6}$, Koichi Watashi ${ }^{7,8}$, Satoshi Yamagoe ${ }^{9}$, Yoshitsugu Miyazaki ${ }^{9}$, Takaji Wakita', Koichi Izumikawa ${ }^{3}$, Katsunori Yanagihara ${ }^{10}$, Hiroshi Mukae 2,11 , Shigeru Kohno ${ }^{2}$ and Nelfinavir Study Group
Abstract
Objectives: The aim of this trial is to evaluate the antiviral efficacy, clinical efficacy, and safety of nelfinavir in patients with asymptomatic and mild COVID-19.
Trial design: The study is designed as a multicenter, open-label, blinded outcome assessment, parallel group, investigator-initiated, exploratory, randomized (1:1 ratio) controlled clinical trial.
Participants: Asymptomatic and mild COVID-19 patients will be enrolled in 10 university and teaching hospitals in Japan. The inclusion and exclusion criteria are as follows:
Inclusion criteria:
(1) Japanese male or female patients aged $\geq 20$ years
(2) SARS-CoV-2 detected from a respiratory tract specimen (e.g., nasopharyngeal swab or saliva) using PCR, LAMP,
or an antigen test within 3 days before obtaining the informed consent
(3) Provide informed consent

Exclusion criteria:

(1) Symptoms developed $\geq 8$ days prior to enrolment

(Continued on next page)

\footnotetext{
* Correspondence: taiga-m@nagasaki-u.ac.jp

2Department of Respiratory Medicine, Nagasaki University Hospital, Nagasaki,

Japan

${ }^{3}$ Department of Infectious Diseases, Nagasaki University Graduate School of

Biomedical Sciences, 1-7-1 Sakamoto, Nagasaki 852-8501, Japan

Full list of author information is available at the end of the article
}

(c) The Author(s). 2021 Open Access This article is licensed under a Creative Commons Attribution 4.0 International License, which permits use, sharing, adaptation, distribution and reproduction in any medium or format, as long as you give appropriate credit to the original author(s) and the source, provide a link to the Creative Commons licence, and indicate if changes were made. The images or other third party material in this article are included in the article's Creative Commons licence, unless indicated otherwise in a credit line to the material. If material is not included in the article's Creative Commons licence and your intended use is not permitted by statutory regulation or exceeds the permitted use, you will need to obtain permission directly from the copyright holder. To view a copy of this licence, visit http://creativecommons.org/licenses/by/4.0/ The Creative Commons Public Domain Dedication waiver (http://creativecommons.org/publicdomain/zero/1.0/) applies to the data made available in this article, unless otherwise stated in a credit line to the data. 
(Continued from previous page)

(2) $\mathrm{SpO}_{2}<96 \%$ (room air)

(3) Any of the following screening criteria:

a) $\mathrm{ALT}$ or $\mathrm{AST} \geq 5 \times$ upper limit of the reference range

b) Child-Pugh class B or C

c) Serum creatinine $\geq 2 \times$ upper limit of the reference range and creatinine clearance $<30 \mathrm{~mL} / \mathrm{min}$

(4) Poorly controlled diabetes (random blood glucose $\geq 200 \mathrm{mg} / \mathrm{dL}$ or HbA1c $\geq 7.0 \%$, despite treatment)

(5) Unsuitable serious complications based on the assessment of either the principal investigator or the subinvestigator

(6) Hemophiliac or patients with a marked hemorrhagic tendency

(7) Severe diarrhea

(8) Hypersensitivity to the investigational drug

(9) Breastfeeding or pregnancy

(10)With childbearing potential and rejecting contraceptive methods during the study period from the initial administration of the investigational drug

(11)Receiving rifampicin within the previous 2 weeks

(12)Participated in other clinical trials and received drugs within the previous 12 weeks

(13)Undergoing treatment for HIV infection

(14)History of SARS-CoV-2 vaccination or wishes to be vaccinated against SARS-CoV-2

(15)Deemed inappropriate (for miscellaneous reasons) based on the assessment of either the principal investigator or the sub-investigator

Intervention and comparator: Patients who meet the inclusion criteria and do not meet any of the exclusion criteria will be randomized to either the nelfinavir group or the symptomatic treatment group.

The nelfinavir group will be administered $750 \mathrm{mg}$ of nelfinavir orally, three times daily for 14 days (treatment period). However, if a participant tests negative on two consecutive PCR tests of saliva samples, administration of the investigational drug for that participant can be discontinued at the discretion of the investigators.

The symptomatic treatment group will not be administered the investigational drug, but all other study procedures and conditions will be the same for both groups for the duration of the treatment period. After the treatment period of 14 days, each group will be followed up for 14 days (observational period).

Main outcomes: The primary endpoint is the time to negative conversion of SARS-CoV-2. During the study period from Day 1 to Day 28, two consecutive negative PCR results of saliva samples will be considered as the negative conversion of the virus.

The secondary efficacy endpoints are as follows:

For patients with both asymptomatic and mild disease: area under the curve of viral load, half decay period of viral load, body temperature at each time point, all-cause mortality, incidence rate of pneumonia, percentage of patients with newly developed pneumonia, rate of oxygen administration, and the percentage of patients who require oxygen administration.

For asymptomatic patients: incidence of symptomatic COVID-19, incidence of fever $\left(\geq 37.0^{\circ} \mathrm{C}\right.$ for two consecutive days), incidence of cough

For patients with mild disease: incidence of defervescence $\left(<37.0^{\circ} \mathrm{C}\right)$, incidence of recovery from clinical symptoms, incidence of improvement of each symptom

The secondary safety endpoints are adverse events and clinical examinations.

Randomization: Patients will be randomized to either the nelfinavir group or the symptomatic treatment group using the electric data capture system (1:1 ratio, dynamic allocation based on severity [asymptomatic], and age $[<$ 60 years]).

Blinding (masking): Only the assessors of the primary outcome will be blinded (blinded outcome assessment). (Continued on next page) 
(Continued from previous page)

Numbers to be randomized (sample size): The sample size was determined based on our power analysis to reject the null hypothesis, $\mathrm{S}(\mathrm{t} \mid \mathrm{z}=1)=\mathrm{S}(\mathrm{t} \mid \mathrm{z}=0)$ where $\mathrm{S}$ is a survival function, $\mathrm{t}$ is time to negative conversion, and $z$ denotes randomization group, by the log-rank test with a two-sided $p$ value of 0.05 . We estimated viral dynamic parameters by fitting a nonlinear mixed-effects model to reported viral load data, and simulated our primary endpoint from viral-load time-courses that were realized from sets of viral dynamics parameters sampled from the estimated probability distribution of the parameters (sample size: 2000; 1000 each for randomization group). From this estimation of the hazard ratio between the randomization groups for the event of negative conversion using this simulation dataset, the required number of events for rejecting our null hypothesis with a power of 0.80 felled 97.345 by plugging the estimated hazard ratio, 1.79, in Freedman's equation. Therefore, we decided the required number of randomizations to be 120 after consideration of the frequency of censoring and the anticipated rate of withdrawal caused by factors such as withdrawal of consent.

Trial Status: Protocol version 6.0 of February 12, 2021. Recruitment started on July 22, 2020 and is anticipated to be completed by March 31, 2022.

Trial registration: This trial was registered in Japan Registry of Clinical Trials (jRCT) (jRCT2071200023) on 21 July 21 , 2020.

Full protocol: The full protocol is attached as an additional file, accessible from the Trials website (Additional file 1). In the interest in expediting dissemination of this material, the familiar formatting has been eliminated; this Letter serves as a summary of the key elements of the full protocol.

The study protocol has been reported in accordance with the Standard Protocol Items: Recommendations for Clinical Interventional Trials (SPIRIT) guidelines (Additional file 2).

Keywords: nelfinavir, COVID-19, SARS-CoV-2, asymptomatic, mild, blinded outcome assessment, randomized controlled trial, protocol

\section{Supplementary Information}

The online version contains supplementary material available at https://doi. org/10.1186/s13063-021-05282-w.

Additional file 1. Full study protocol.

Additional file 2. SPIRIT 2013 Checklist: Recommended items to address in a clinical trial protocol and related documents*.

\section{Acknowledgements}

We would like to thank the Nelfinavir Study Group, in which principal investigators of the study sites are as follows: Hiroshi Yotsuyanagi (IMSUT Hospital of The Institute of Medical Science, The University of Tokyo), Yasuyuki Kato and Tetsuya Matsumoto (International University of Health and Welfare Narita Hospital), Norihito Tarumoto (Saitama Medical University Hospital), Satoshi Shiraishi (Osaka City Juso Hospital), Hiroshi Ishii (Fukuoka University Chikushi Hospital), Hideaki Ohno (Saitama Medical Center), Kazuhiro Yatera (Hospital of the University of Occupational and Environmental Health, Japan), Yoshihiro Yamamoto (Toyama University Hospital ), and Hiroshi Kakeya (Osaka City University Hospital).

\section{Authors' contributions \\ All authors participated in the methodology and acquisition of this protocol summary and have given their final approval of this manuscript to be published as presented. NH, TM, HY, SK, SI, KW, YM, and TW were involved in the design of this trial. SM, SI, and YK are involved in the formal analysis. $\mathrm{NH}$, TM, Y Fukushige, ST, MH, TK, and $\mathrm{HH}$ are involved in project administration. $\mathrm{TM}, \mathrm{Y}$ Fujii, and $\mathrm{MH}$ are involved in data curation and validation of the study results.}

\section{Funding}

The study is funded by the Health and Labor Sciences Research Grants program (grant no.: 20HA1008). The investigational drug (nelfinavir) and the nelfinavir drug information are provided by Japan Tobacco Inc. and Pfizer Inc. The funding body and pharmaceutical companies have had no role in the design of the study and will have no role in the collection, analysis, and interpretation of data and in writing the manuscript of the primary study results.

\section{Availability of data and materials}

The full study protocol is available in the supplementary materials and at https://jrct.niph.go.jp/en-latest-detail/jRCT2071200023. The data are not available because the trial is in progress. The data will be made available from the author on reasonable request once the trial has been completed. Please contact the corresponding author, Dr. T. Miyazaki (taiga-m@nagasakiu.ac.jp).

\section{Declarations}

\section{Ethics approval and consent to participate}

The protocol was approved by the Nagasaki University Hospital Institutional Review Board (approval number: I20-001) on May 25, 2020, and by each local Institutional Review Board. The authors certify that this trial has received ethical approval from the appropriate ethics committees of all the study sites. Participants will be provided with the information regarding the study by their respective infectious disease specialists and requested to sign an informed consent form if they are willing to participate. Participants should have the ability to understand and be willing to sign a written informed consent document to accept randomization to either intervention arm.

\section{Consent for publication}

Not applicable.

\section{Competing interests}

$\mathrm{TM}, \mathrm{KI}, \mathrm{KY}, \mathrm{HM}$, and SK have received lecture honoraria and research grants from Pfizer Inc. outside the submitted work. The other authors declare that they have no competing interests.

\section{Author details}

${ }^{1}$ Clinical Research Center, Nagasaki University Hospital, Nagasaki, Japan. ${ }^{2}$ Department of Respiratory Medicine, Nagasaki University Hospital, Nagasaki, Japan. ${ }^{3}$ Department of Infectious Diseases, Nagasaki University Graduate 
School of Biomedical Sciences, 1-7-1 Sakamoto, Nagasaki 852-8501, Japan. ${ }^{4}$ Department of Hospital Pharmacy Nagasaki University Hospital, Nagasaki, Japan. ${ }^{5}$ Clinical Research Center, Chiba University Hospital, Chiba, Japan. ${ }^{6}$ Department of Biology, Faculty of Sciences, Kyushu University, Fukuoka, Japan. ${ }^{7}$ Department of Virology II, National Institute of Infectious Diseases, Tokyo, Japan. ${ }^{8}$ Research Center for Drug and Vaccine Development, National Institute of Infectious Diseases, Tokyo, Japan. ${ }^{9}$ Department of Chemotherapy and Mycoses, National Institute of Infectious Diseases, Tokyo, Japan.

${ }^{10}$ Department of Laboratory Medicine, Nagasaki University Hospital, Nagasaki, Japan. "Department of Respiratory Medicine, Nagasaki University Graduate School of Biomedical Sciences, Nagasaki, Japan.

Received: 16 April 2021 Accepted: 17 April 2021

Published online: 28 April 2021

\section{Publisher's Note}

Springer Nature remains neutral with regard to jurisdictional claims in published maps and institutional affiliations.

Ready to submit your research? Choose BMC and benefit from:

- fast, convenient online submission

- thorough peer review by experienced researchers in your field

- rapid publication on acceptance

- support for research data, including large and complex data types

- gold Open Access which fosters wider collaboration and increased citations

- maximum visibility for your research: over $100 \mathrm{M}$ website views per year

At BMC, research is always in progress.

Learn more biomedcentral.com/submissions 